\title{
PROGRAMA BOLSA FAMÍLIA E MORTALIDADE INFANTIL NO BRASIL: REVISÃO INTEGRATIVA
}

\author{
E. S. A. SILVA ${ }^{1,}$, N. A. PAES ${ }^{2}$ \\ ${ }^{1,2}$ Universidade Federal da Paraíba, Departamento de Estatística \\ everlanesuane@hotmail.com ${ }^{1}$
}

Submetido 15/07/2016 - Aceito em 25/01/2018

DOI: $10.15628 /$ holos.2018.4836

\section{RESUMO}

Objetivo. Revisar a literatura a respeito do impacto do Programa Bolsa Família (PBF) sobre a mortalidade infantil no Brasil. Métodos. Foi realizada uma revisão integrativa para responder ao objetivo proposto. Foram consultadas as bases de dados Literatura LatinoAmericana e do Caribe em Ciências da Saúde (LILACS) e PubMed além das bibliotecas eletrônicas Portal de Periódicos CAPES/MEC, Scientific Electronic Library Online (SciELO) e Revisões Sistemáticas da Colaboração Cochrane (Cochrane), durante o período de junho a agosto de 2014. As seguintes etapas foram realizadas: identificação do problema de revisão, formulação da questão norteadora, seleção da amostra, categorização e análise dos dados, discussão dos resultados e síntese do conhecimento. Resultados. Foram identificados 1527 artigos. Após a aplicação dos critérios de inclusão e exclusão, 3 artigos integraram o estudo. Os estudos selecionados enfatizam que o PBF contribuiu de forma positiva e substancial para a queda das Taxas de Mortalidade Infantil no Brasil. Entretanto, alertam para a importância dos efeitos combinados do PBF junto ao Estratégia Saúde da Família. Conclusões. O PBF contribuiu com decréscimos relevantes nos níveis da mortalidade infantil. Para a manutenção desta redução faz-se necessário intervenções governamentais que minimizem as disparidades de renda experimentada por grande parte do povo brasileiro, e que os gestores disponibilizem meios para cumprimento de maneira adequada das condicionalidades impostas aos beneficiários do programa.

\section{THE BOLSA FAMÍLIA CASH TRANSFER PROGRAM AND INFANT MORTALITY IN BRAZIL: AN INTEGRATIVE REVIEW}

\begin{abstract}
Objective. To review the literature regarding the impact of Programa Bolsa Família (PBF) on infant mortality in Brazil. Methods. We carried out an integrative review to meet the proposed objective. The Cochrane Library, LILACS, PubMed, journals website CAPES/MEC and SciELO databases, as well as public organization websites were searched from June to August 2014. The following steps were taken: identification of the problem, formulation of guiding question, sample selection, categorization and analysis of data, discussion of results and synthesis of knowledge. Results. 1527 articles were identified. After applying the inclusion and exclusion criteria, three studies were included in this
\end{abstract}

review. They emphasize that since the creation of the $\mathrm{PBF}$, it has contributed positively and significantly to the decline of infant mortality rates in Brazil. However, they highlight the importance of the combined effects of PBF with the Estratégia Saúde da Família Program in the struggle for this decline. Conclusions. The PBF contributed significantly to the decrease in infant mortality. For the maintenance of this reduction is recomended necessary government intervention to minimize disparities in income experienced by the much of the Brazilian people, and that managers produce adequate means to fulfill adequately the conditionalities imposed on program beneficiaries.

KEYWORDS: Infant mortality, Programa Bolsa Família, Income transfer programs, Brazil. 


\section{INTRODUÇÃO}

Os Programas de transferência condicional de renda surgiram buscando romper o ciclo intergeracional da pobreza, fornecendo uma fonte de renda regular mínima para famílias pobres que é "condicional" em seu investimento na saúde e educação de seus filhos (SHEI, 2013). A preocupação com a transferência de renda no mundo é histórica. Existem diversas gerações de programas de transferências de renda, onde a primeira delas começou na América Latina no final do século XX, concentrando suas ações nas áreas de saúde e educação (BASSETT, 2008; MATTEI, 2010).

O Programa Bolsa Família (PBF) do Brasil consiste na transferência direta de renda que beneficia famílias em situação de pobreza e de extrema pobreza, criado em 2003 e considerado o programa de transferência monetária de maior envergadura no mundo (MDSA, 2013; RASELLA et al., 2013; WOLF \& BARROS FILHO, 2014). Os benefícios são específicos para famílias com crianças, jovens até 17 anos, gestantes e mães que amamentam (MDSA, 2013).

Até meados de agosto de 2014, o PBF já tinha beneficiado, aproximadamente, 14 milhões de famílias em todos os municípios brasileiros (MDSA, 2013). Levando-se em conta os valores investidos neste programa, torna-se imperioso avaliar sua eficiência e efetividade, situação que pode ser extrapolada a outros Países (WOLF \& BARROS FILHO, 2014). Quase todos os Países da América Latina têm um programa deste tipo. Eles também existem em Países asiáticos como Bangladesh, Indonésia, Malawi e Turquia (MDSA, 2013). A importância e impacto desses programas no combate a pobreza têm despertado interesse mesmo em alguns Países desenvolvidos (MCCOLL, 2008; SHEI, 2013).

As questões relacionadas à pobreza são persistentes e recorrentes, onde inúmeros estudos buscam avaliar os efeitos deste fenômeno (GILLESPIE et al., 1996; SMITH \& HADDAD, 2000; MONTEIRO et al., 2002; MONTEIRO, 2003).

De acordo com as normas vigentes em 2014, o PBF brasileiro oferece às famílias quatro tipos de benefícios: básico, variável, variável vinculado ao adolescente e variável de caráter extraordinário (MDSA, 2013).

As principais condicionalidades do PBF são: vacinação conforme o calendário vacinal e acompanhamento do crescimento e desenvolvimento das crianças menores de 7 anos, acompanhamento da saúde das mulheres na faixa de 14 a 44 anos e de gestantes e nutrizes. Na educação, todas as crianças e adolescentes entre 6 e 17 anos devem apresentar a frequência escolar mensal mínima exigida (COTTA \& MACHADO, 2013).

Merece relevo a velocidade de implementação do PBF, retratada pelos dados de cobertura. Em dezembro de 2003, o Programa atendia 3,6 milhões de famílias, passando para 6,5 milhões no mesmo mês do ano seguinte e, em dezembro de 2005 , alcançou um total de 8,7 milhões. No início de 2006, o PBF já atingia a meta prevista de 11,1 milhões de famílias atendidas (SENNA et al., 2007). Ao todo, em junho de 2014, 36 milhões de brasileiros estavam fora da extrema pobreza em decorrência da transferência de renda (MDSA, 2013).

O primeiro aspecto tratado pelo Artigo 227 da Constituição Federal brasileira é assegurar à criança o direito à vida. Deste modo, todo o empenho da família, sociedade e do Estado deve ser prioritariamente em prol da luta pelo afastamento da morte, ou seja, pela redução ou eliminação da mortalidade infantil (CONSTITUIÇÃO, 1988).

Durante a década de 1960, os níveis de mortalidade infantil se estabilizaram no Brasil, em praticamente todas as regiões. Na década de 1970, a mortalidade infantil ultrapassou 90 óbitos por 1.000 nascidos vivos, onde considerável parcela desses óbitos foi atribuída a causas passíveis de prevenção, principalmente ao binômio infecção-desnutrição. Já em 1990, a Taxa de 
Mortalidade Infantil era de 48,3 óbitos por 1.000 nascidos vivos, diminuindo ainda mais na década seguinte, chegando em 2009 a 14,8 óbitos de menores de um ano por 1.000 nascidos vivos (IBGE, 1999; MOREIRA et al., 2012).

Pelo menos desde os anos 1970, a mortalidade infantil é vista como uma ocorrência "evitável" por serviços de saúde eficazes, constituindo-se em um "evento sentinela" da qualidade da atenção médica (RUTSTEIN et al., 1976; HARTZ et al., 1996). O Brasil atingiu a meta da Organização das Nações Unidas (ONU) de reduzir em dois terços os indicadores de mortalidade de crianças de até cinco anos, no período de 1990 a 2015. Ou seja, a mortalidade de crianças de até cinco anos caiu de 53,7 óbitos por mil nascimentos, em 1990, para 17,7 em 2011 (IPEA, 2014). As Taxas de Mortalidade Infantil já estavam diminuindo antes do início do Bolsa Família, mas a taxa de declínio parece ter aumentado após a implementação do programa (SHEI, 2013).

Existem diferenças substanciais e preocupantes nas Taxas de Mortalidade Infantil entre grupos populacionais, além de que um grande número de mortes afetam principalmente as regiões mais desfavorecidas da sociedade, passíveis de serem evitadas, por meio de programas preventivos, como o PBF (SIMÕES, 2002; UNICEF, 2008).

Dado este contexto, teve-se como objetivo neste estudo revisar a literatura a respeito do impacto do Programa Bolsa Família no contexto da mortalidade infantil no Brasil. Almeja-se, assim, tratar dessa relação de causa e efeito, bem como subsidiar investigações futuras e descrever o atual estado da arte relativo a esta temática.

\section{METODOLOGIA}

Trata-se de um estudo bibliográfico, tipo Revisão Integrativa da Literatura, o qual possibilita a busca, a avaliação crítica e a síntese de estudos já publicados em relação ao tema investigado (MENDES et al., 2008; POMPEO et al., 2009).

Para tanto, as seguintes etapas foram realizadas: 1-identificação do problema de revisão, 2-formulação da questão norteadora, 3-seleção da amostra, 4-categorização e análise dos dados, 5-discussão dos resultados e síntese do conhecimento (WHTTEMORE \& KNAFF, 2005).

Foi definida como questão norteadora da pesquisa: "Qual o impacto do Programa Bolsa Família sobre a mortalidade infantil?". A coleta dos dados foi realizada no período de junho a agosto de 2014, através de pesquisas nas bases de dados eletrônicas da Literatura Latino Americana e do Caribe (LILACS), PubMed, Portal de Periódicos CAPES/MEC, Scientific Electronic Library Online (SciELO) e Biblioteca Cochrane. Foram adotados descritores e palavras combinadas nas línguas inglesa, portuguesa e espanhola: "mortalidade infantil", "Brasil", "Bolsa Família", "programa de transferência de renda" e "programas governamentais". A busca utilizou os termos individualmente e cruzados, recorrendo ao operador booleano AND: Government Programs AND Infant Mortality; Cash Transfer Program AND Mortality; Government Programs AND Infant Mortality AND Brazil; Cash AND Program AND Transfer AND Mortality; Programas de Gobierno AND Mortalidad Infantil; Bolsa Família.

Os artigos localizados foram submetidos aos seguintes critérios de inclusão: artigos completos e disponíveis com acesso livre, publicados entre 2003 e 2014, idiomas já citados e abordagem do impacto do Programa Bolsa Família sobre a mortalidade infantil. Foram excluídos artigos repetidos nas bases de dados, revisões integrativas e revisões sistemáticas, estudos de caso ou comunicação e trabalhos com resultados insuficientes ou confusos.

Os estudos foram selecionados utilizando os critérios de inclusão e exclusão, e ao realizar uma leitura prévia foram selecionados aqueles com conteúdo relevante para o objeto de estudo e discussão acerca da problemática em pauta. 
Após a leitura dos estudos foram extraídas as informações mais relevantes relacionadas à associação entre Programa Bolsa Família e mortalidade infantil. Os estudos foram desenvolvidos com base em dados secundários e abordam o impacto do programa de transferência condicional de renda Bolsa Família na mortalidade infantil no Brasil desde sua implantação.

\section{RESULTADOS E DISCUSSÃO}

Foram identificados 1527 artigos através da busca nas bases de dados eletrônicas (LILACS, PubMed, Portal de Periódicos CAPES/MEC, SciELO e Biblioteca Cochrane) utilizando a combinação dos descritores e palavras-chave. Em seguida, foi realizada uma filtragem onde foram excluídos 1524 estudos por não atenderem aos critérios de inclusão, resultando ao final em apenas 3 estudos que abordaram a temática pertinente à revisão.

O processo de filtragem dos trabalhos através do cruzamento dos descritores e palavraschave por bases de dados são descritos na Tabela 1. Todos os títulos dos artigos selecionados apresentavam como enfoque a mortalidade infantil e o Programa Bolsa Família. Em relação à Biblioteca ou base de dados pesquisada, a PubMed exibiu os 3 estudos selecionados para a revisão.

Tabela 1: Resultado da filtragem para a seleção dos estudos, Brasil, 2004 a 2015.

\begin{tabular}{|c|c|c|c|c|}
\hline $\begin{array}{l}\text { Base de } \\
\text { dados }\end{array}$ & Descritores e palavras-chave & $\begin{array}{l}\text { Estudos } \\
\text { encontrados }\end{array}$ & $\begin{array}{l}\text { Estudos } \\
\text { excluídos }\end{array}$ & $\begin{array}{l}\text { Total de estudos } \\
\text { selecionados }\end{array}$ \\
\hline LILACS & $\begin{array}{c}\text { Government Programs AND Infant } \\
\text { Mortality }\end{array}$ & 8 & 8 & 0 \\
\hline LILACS & $\begin{array}{c}\text { Programas de Gobierno AND } \\
\text { Mortalidad Infantil }\end{array}$ & 6 & 6 & 0 \\
\hline PubMed & $\begin{array}{c}\text { Government Programs AND Infant } \\
\text { Mortality }\end{array}$ & 119 & 119 & 0 \\
\hline PubMed & $\begin{array}{c}\text { Cash AND Program AND Transfer } \\
\text { AND Mortality }\end{array}$ & 10 & 8 & 2 \\
\hline PubMed & Bolsa Família & 40 & 39 & 1 \\
\hline SCIELO & $\begin{array}{c}\text { Government Programs AND Infant } \\
\text { Mortality AND Brazil }\end{array}$ & 1270 & 1270 & 0 \\
\hline $\begin{array}{l}\text { Periódicos } \\
\text { Capes }\end{array}$ & $\begin{array}{c}\text { Government Programs AND Infant } \\
\text { Mortality AND Brazil }\end{array}$ & 27 & 27 & 0 \\
\hline $\begin{array}{l}\text { Cochrane } \\
\text { (BVS) }\end{array}$ & $\begin{array}{c}\text { Government Programs AND Infant } \\
\text { Mortality }\end{array}$ & 43 & 43 & 0 \\
\hline $\begin{array}{c}\text { Cochrane } \\
\text { (BVS) }\end{array}$ & $\begin{array}{c}\text { Cash AND Program AND Transfer } \\
\text { AND Mortality }\end{array}$ & 4 & 4 & 0 \\
\hline & TOTAL DE ARTIGOS & 1527 & 1524 & 3 \\
\hline
\end{tabular}

Fonte: Bases de dados eletrônicas.

Para filtrar os estudos que abordassem o tema PBF e mortalidade infantil foi realizada inicialmente a leitura dos títulos dos artigos como primeiro critério para seleção, depois foram 
avaliados os resumos ou abstracts. Em seguida, os artigos foram analisados na íntegra como critério final para inclusão no estudo.

A amostra final desta revisão foi composta por artigos publicados em 2013. Após a seleção dos estudos, todos foram avaliados de acordo com as seguintes variáveis: autores, título do estudo, periódico e ano de publicação, unidade de análise, objetivo e conclusão dos estudos.

As características dos artigos incluídos na seleção final são apresentadas na Tabela 2. Todos eles utilizaram a abordagem metodológica quantitativa e o período global de referência variou de 1998 a 2010. Os estudos selecionados foram publicados em periódicos internacionais. Quanto à abrangência geográfica, dois artigos (GUANAIS, 2013; RASELLA et al., 2013) utilizaram uma amostra dos municípios brasileiros e Amie Shei (2013) considerou todos os municípios do Brasil para o estudo.

Tabela 2: Caracterização dos artigos sobre o Programa Bolsa Família e mortalidade infantil, Brasil, 2004 a 2015.

\begin{tabular}{|c|c|c|c|c|c|}
\hline Autoria & Título do Estudo & $\begin{array}{c}\text { Periódico/Ano } \\
\text { de } \\
\text { Publicação }\end{array}$ & $\begin{array}{l}\text { Unidade de } \\
\text { Análise } \\
\text { (municípios } \\
\text { do Brasil) }\end{array}$ & Objetivo & $\begin{array}{c}\text { Impacto do } \\
\text { Programa Bolsa } \\
\text { Família sobre a } \\
\text { Mortalidade } \\
\text { Infantil }\end{array}$ \\
\hline Rasella et : & $\begin{array}{l}\text { Effect of a conditional } \\
\text { cash transfer } \\
\text { programme on } \\
\text { childhood mortality: a } \\
\text { Nationwide analysis } \\
\text { of Brazilian } \\
\text { municipalities }\end{array}$ & $\begin{array}{c}\text { The Lancet } \\
\text { (2013) }\end{array}$ & 2.853 & $\begin{array}{l}\text { Avaliar o impacto do PBF } \\
\text { sobre a } \mathrm{TM}^{\mathrm{a}} \quad(<5 \text { anos), } \\
\text { centrados nas causas de } \\
\text { morte } \\
\text { diarreia e infecções das } \\
\text { vias } \\
\text { inferiores) e em potenciais } \\
\text { mecanismos (vacina, pré- } \\
\text { natal e internação } \\
\text { hospitalar). }\end{array}$ & Positivo \\
\hline Amie Shei & $\begin{array}{l}\text { Brazil's Conditional } \\
\text { Cash Transfer } \\
\text { Program Associated } \\
\text { with Declines in Infant } \\
\text { Mortality Rates }\end{array}$ & $\begin{array}{l}\text { Health Affairs } \\
\qquad(2013)\end{array}$ & 5.564 & $\begin{array}{l}\text { Avaliar se as diferenças } \\
\text { anuais das taxas de } \\
\text { mortalidade infantil eram } \\
\text { associadas às alterações } \\
\text { anuais da cobertura do } \\
\text { Programa Bolsa Família. }\end{array}$ & Positivo \\
\hline Guanais & $\begin{array}{l}\text { The Combined Effects } \\
\text { of the Expansion of } \\
\text { Primary Health Care } \\
\text { and Conditional Cash } \\
\text { Transfers on Infant } \\
\text { Mortality in Brazil, } \\
\text { 1998-2010 }\end{array}$ & $\begin{array}{l}\text { American Journal } \\
\text { of Public Health } \\
\qquad(2013)\end{array}$ & 4.583 & $\begin{array}{l}\text { Examinar os efeitos } \\
\text { combinados do Programa } \\
\text { de Saúde da Família (PSF) } \\
\text { e do Programa Bolsa } \\
\text { Família (PBF) sobre a } \\
\text { mortalidade infantil pós- } \\
\text { neonatal (PNIM) no Brasil. }\end{array}$ & Positivo \\
\hline
\end{tabular}

Fonte: Artigos inclusos na revisão integrativa. $\mathrm{TM}^{\mathrm{a}}$ - Taxa de Mortalidade 
Rasella et al. (2013) avaliaram, utilizando fontes do governo, o efeito do PBF em mortes de crianças menores de 5 anos, em geral, associadas à pobreza: desnutrição, diarreia e infecções respiratórias inferiores. O período de referência coberto pelo estudo compreende de 2004-2009 e inclui apenas municípios brasileiros considerados com estatísticas vitais de qualidade adequada (óbitos e registro de nascimento).

Nesse estudo foram utilizados modelos de regressão negativa binomial para dados em painel com efeitos fixos e ajustados para covariáveis que expressaram relevantes aspectos sociais e econômicos, bem como o efeito do maior programa de cuidados primários da saúde no País (Estratégia Saúde da Família - ESF). Os autores concluíram que as taxas de mortalidade em menores de cinco anos, geral e por causas relacionadas com a pobreza, diminuíram com o aumento da cobertura do PBF no Brasil.

Amie Shei (2013) usou dados de todos os municípios brasileiros para a análise, decompõe a mortalidade infantil em neonatal (número de óbitos durante os primeiros 28 dias de vida por 1.000 nascidos vivos) e pós-neonatal (número de mortes depois de 28 dias de vida, mas antes de um ano de vida para cada 1.000 nascidos vivos). O estudo considera como referência o período 1998-2008. Foi utilizado como método de análise os modelos de regressão de mínimos quadrados ordinários com efeitos fixos.

Os resultados do estudo revelaram que o PBF foi associado com melhorias significativas na saúde infantil no Brasil, sendo essas melhorias maiores no período pós-neonatal, em municípios com alta cobertura da Estratégia Saúde da Família e os com maiores taxas de mortalidade infantil relacionadas à pobreza.

Guanais (2013) por sua vez, avaliou os efeitos do PBF sobre a mortalidade infantil pósneonatal, justificando a escolha por causa da sua maior receptividade aos cuidados primários de saúde. O estudo longitudinal considerou o período 1998 até 2010 e incluiu 4.583 municípios brasileiros $(82,4 \%$ do total), sendo excluídos 449 municípios localizados na região Norte (indisponibilidade de dados de covariáveis) e outros 533 municípios (apontados em estudos anteriores com baixa qualidade das estatísticas vitais).

O estudo considerou como método de análise a estimação de modelos de regressão de mínimos quadrados ordinários com efeitos fixos. O autor ressaltou a importância do programa brasileiro Estratégia Saúde da Família (ESF) que objetiva ampliar o acesso aos serviços públicos de saúde, especialmente em áreas carentes, oferecendo gratuitamente cuidados de saúde à comunidade (GUANAIS, 2013; RASELLA et al., 2013).

Os estudos se propuseram a mostrar, utilizando diferentes metodologias, que programas de transferência condicional de renda são capazes de gerar resultados satisfatórios à população.

Os resultados desta revisão constataram que o programa de transferência condicional de renda Bolsa Família apresenta um impacto positivo para a redução da mortalidade infantil no Brasil (GUANAIS, 2013; RASELLA et al., 2013; SHEI, 2013). O Censo Demográfico brasileiro (2010) revelou a continuidade de queda do índice de mortalidade infantil. Mas, ao comparar o Brasil com Países desenvolvidos, como Singapura e Japão, que apresentam aproximadamente três mortes por mil nascidos vivos, ou mesmo a alguns Países sul-americanos, como Argentina e Uruguai, equivalente a 13,4 e 13,1, respectivamente, o Brasil ainda precisa avançar muito (MINISTÉRIO DA SAÚDE, 2010; SECRETARIA DE SAÚDE/SP, 2015).

A associação de uma maior cobertura da ESF com uma menor taxa de mortalidade pósneonatal se tornou mais forte quando a cobertura do PBF aumentou (GUANAIS, 2013). Os resultados sugerem que a introdução dos programas de transferência condicional de renda é um fator adicional importante em relação à redução da mortalidade infantil (RASELLA et al., 2013). 
O Fundo das Nações Unidas para a Infância (UNICEF) tem como esfera prioritária de ação a abordagem dos aspectos relacionados ao direito à sobrevivência, nutrição e oferta de serviços básicos capazes de salvar vidas e contribuir para o crescimento saudável das crianças. A Taxa de Mortalidade Infantil é o primeiro indicador sociodemográfico usado pelo UNICEF para medir essa "sobrevivência". Desta forma, iniciativas governamentais que buscam colaborar com as ações protetoras das crianças são vistas como possibilidades de realização dos objetivos dos órgãos apoiadores da infância (IBGE, 2010).

No Brasil, considerando o período entre 2000 e 2010, o número de óbitos de crianças menores de um ano caiu de 29,7 para 15,6 para cada mil nascidas vivas, um decréscimo de 47,6\% na taxa brasileira de mortalidade infantil, sendo a maior queda a do Nordeste, passando de 44,7 para 18,5 óbitos (IBGE, 2005; IBGE, 2012).

O PBF pode afetar a mortalidade infantil através de diferentes caminhos (SHEI, 2013) e é reconhecido pela ONU como bom exemplo de política pública para redução das desigualdades sociais e combate à fome (PORTAL BRASIL, 2014).

A determinação da pobreza e suas consequências sobre a saúde das crianças é de natureza social e coletiva, devendo ser alvo de políticas públicas, mas, os programas exigem compromissos (Condicionalidades) do indivíduo para obtenção do benefício. Em contraste, a renúncia fiscal realizada sobre o pagamento de seguro privado de saúde no Brasil não requer o cumprimento de qualquer obrigatoriedade para as famílias de renda mais alta que dela se beneficiam.

Apesar da pequena expressão numérica dos artigos analisados, os resultados convergiram para a mesma conclusão, propiciando evidências estatísticas sobre esta problemática. Todos os trabalhos foram unânimes em detectar a real importância motivada pelas ações governamentais que objetivam auxiliar o combate de dois agravos importantes no País: pobreza e elevados níveis de mortalidade infantil.

\subsection{Críticas e limitações dos estudos}

Com a disponibilização das bases de dados do PBF nas plataformas do Ministério de Desenvolvimento Social e Combate à Fome - MDS, a partir de 2003 e o reconhecimento da importância dos sistemas de informação para avaliação do impacto do PBF no Brasil, era esperado que houvesse uma crescente produção sobre a utilização desses dados ao nível acadêmico. No entanto, o que se observa é um vasto uso desse impacto na vida das pessoas nos meios de comunicação e com fins políticos.

Se essa produção é incipiente, ela é ausente quando se trata de desagregações espaciais regionais ou áreas específicas do Brasil. Apesar do recurso de busca booleana AND ter contemplado cinco combinações de termos, o total de estudos selecionados não passou de três. Estes trabalhos lançam mão de uma abordagem quantitativa, evidenciando uma lacuna no enfoque qualitativo.

Os três estudos utilizaram dados secundários para análise do impacto trazido pelo Programa Bolsa Família sobre a mortalidade infantil, ou seja, estão sujeitos a erros ligados a qualidade dos dados. Por exemplo, vários municípios brasileiros, ainda, buscam alcançar um nível satisfatório de cobertura dos dados de óbitos. Sendo assim, técnicas de correções adequadas são imprescindíveis para obtenção de dados mais confiáveis para a análise.

Ao considerar o número de estudos analisados e as desigualdades regionais presentes nos diferentes municípios brasileiros, se faz necessário admitir limitações em relação às inferências mais conclusivas neste trabalho, podendo citar também a chance da existência de dados 
negativos ligados ao efeito do PBF que possam não ter sido esclarecidos nos estudos investigados.

É bem certo que o número limitado de artigos pode dificultar uma avaliação mais consistente do conjunto de evidências apresentadas. No entanto, há que se ponderar que não há na literatura referência a um número suficientemente grande para revisões sistemáticas ou integrativas que não incluam metanálise de resultados.

O número reduzido de artigos produzidos durante os doze anos desde a implantação do PBF em 2003 tem demonstrado a dificuldade em sua produção em termos de uso com amplas metodologias estatísticas. Isto revela que, embora a produção nacional tenha crescido em diversos campos da ciência, não é o que se vem observando com esta temática. É preciso notar que dois dos artigos usaram como unidade de análise uma grande quantidade de municípios brasileiros, 2853 (RASELLA et al., 2013) e 4583 (GUANAIS, 2013), e o terceiro, a totalidade deles, ou seja, 5564 (SHEl, 2013).

Ao lidar com uma quantidade enorme de unidades geográficas, os artigos tratados não se descuidaram dos aspectos da qualidade dos dados básicos, cobertura, confiabilidade e incompletitude dos dados e possíveis fontes de vieses. Essas questões foram contempladas à luz dos requerimentos estatísticos impostos pelos sofisticados modelos utilizados, mas não os isentam de possíveis vieses e violações de supostos adicionais reconhecidos pelos próprios autores.

Tratam os três artigos de estudos ecológicos ao nível municipal, sem o uso dos microdados dos beneficiários do PBF. No entanto, é preciso notar que nas bases de dados do PBF as ocorrências dos óbitos das famílias beneficiárias não são disponibilizadas, o que impede verificar essa relação diretamente a partir dos microdados. Por se tratarem de estudos ecológicos, pode ser que as pessoas beneficiárias não sejam as mesmas pessoas que estão experimentando os benefícios na saúde ou que os nascimentos decorrentes dos pais beneficiários tenham vindo a óbito. Em alguns casos, como no primeiro estudo (GUANAIS, 2013), a exclusão de municípios de algumas regiões, como a Norte do País introduz algum tipo de viés nos resultados, desde que esta região é uma das grandes beneficiárias do Programa. Sem embargo, esta exclusão pode ter resultado em uma subestimação do impacto do Programa, já que esta região juntamente com a do Nordeste têm as maiores taxas de beneficiários e são mais prováveis de serem beneficiadas pela expansão da saúde primária e das transferências de renda.

Outra questão diz respeito às comparações municipais com grandes variações em seu tamanho populacional. Uma abordagem mais comparável seria dividir as populações por seu porte populacional, por exemplo, de acordo com os recortes geográficos adotados pelo IBGE em seus estudos regionalizados: municípios de porte baixo, médio, grande e metrópoles. Muito embora, o estudo de Guanais (2013) tenha ponderado os municípios pelo seu porte populacional, este artifício, no entanto, pode não superar o problema da qualidade com os dados (cobertura, confiabilidade, regularidade) para os pequenos municípios, os quais são mais susceptíveis a erros de registros inadequados.

O PBF pode ainda ter sido adotado em municípios onde a mortalidade pós-neonatal já estava declinando por outras ações públicas antes mesmo dele ser implantado. Desta forma, o efeito direto do PBF pode ser também creditado a estas ações, como a ESF e mesmo a outras ações sem a presença desta última.

Estudos ecológicos não permitem verificar como o Programa pode afetar a saúde. Neste caso, o PBF é conduzido pelo Ministério de Desenvolvimento Social e Agrário e não pelo Ministério da Saúde, o que sugere que sua adoção é independente das condições de saúde dos municípios. 
A TMI já estava em declínio décadas antes do PBF ser implantado em 2003, porém ele ajudou a acelerar esta redução. Para Amie Shei (2013), o efeito na redução na mortalidade infantil até 2008 foi de $9,3 \%$ e de $24,3 \%$ na mortalidade pós-neonatal, maior que na neonatal. Este estudo leva a indagar sobre a meta de redução da mortalidade que o programa de transferência de renda pretende alçar e se efetivamente a alcançou. Não há clareza sobre esta questão. No entanto, é possível dizer que o Brasil cumpriu antes do tempo a meta de redução da mortalidade infantil que fez parte dos oito Objetivos de Desenvolvimento do Milênio (ODM) delineados pela Organização das Nações Unidas (ONU), ao analisar os maiores problemas mundiais em 2000. Neste ano, a meta era de alcançar 15,7 óbitos infantis por mil nascidos vivos em 2015. Em 2000 a TMI era de 47,1 e em 2014 de 15,3 (IPEA, 2014), valor este abaixo da meta.

Com a manutenção da cobertura do PBF e da ESF, é possível que a redução na TMI continue. No entanto, até atingir os níveis de Países mais avançados como a do Japão e Suécia, em torno de $2 \%$, por exemplo, ainda há um longo caminho a percorrer. Assim, programas como - Bolsa Família ainda são capazes de fazer a diferença na manutenção da redução dos níveis da mortalidade infantil no Brasil e, em especial, nas regiões do Norte e Nordeste.

\subsection{Implicações políticas}

Dada a importância do PBF na redução da mortalidade infantil, alguns pontos deveriam ser considerados. Os gestores necessitam examinar a adequação da infraestrutura de saúde nos municípios aos programas de transferência de renda condicionais, uma vez que, a expansão dos programas depende da disponibilidade dos serviços oferecidos pelos sistemas de saúde, devido à imposição das condicionalidades aos beneficiários.

O PBF guarda um grande potencial para reduzir não somente os níveis da mortalidade infantil, mas também para melhorar a saúde da população, particularmente se fortalecesse a infraestrutura de serviços de atenção à saúde, complementasse os serviços de atenção existente como a ESF, e focasse nos grupos vulneráveis aos maiores riscos, não apenas aos de baixa renda.

\subsection{Sugestões para futuras investigações}

A mortalidade neonatal é fortemente associada com a qualidade dos serviços hospitalares prestados aos recém-nascidos, embora a qualidade destes serviços esteja fora do escopo de atuação dos programas o PBF e a ESF.

Sem embargo, estudos sobre as tendências da mortalidade neonatal são necessários como subsídios para os desenhos de políticas e do planejamento para assegurar um progressivo ganho na redução da mortalidade infantil no Brasil.

Pesquisas futuras deveriam examinar os potenciais mecanismos em ação nos programas de transferência de renda através da utilização de microdados ou pesquisas com desenhos amostrais e não somente de dados agregados. Estudos com recortes espaciais estratificados são importantes para se conhecer melhor as diferenciações regionais em que poderiam ser evidenciados os efeitos do PBF na mortalidade infantil. Desta maneira, a alocação dos recursos poderia ser melhor otimizada.

Estudos que avaliem o efeito combinado do PBF com a ESF ainda são raros. Na verdade, o único estudo realizado até esta revisão foi o de Guanais (2013). É pertinente indagar o porquê de tal escassez, já que se tratam dos dois mais importantes programas brasileiros que exercem uma influência direta na melhoria dos níveis da mortalidade infantil no Brasil, implantados em 1994 (ESF) e em 2003 (PBF). Estudos adicionais nesta perspectiva ajudariam a responder a lacunas na literatura e trariam beneficiosos insumos aos gestores sobre estes programas. 


\section{CONCLUSÃO}

Os resultados convergiram para a mesma conclusão ao destacar que o PBF é uma questão relevante de saúde pública e que contribuiu com decréscimos relevantes nos níveis da mortalidade infantil. As evidências estatísticas foram claras ao detectar a real importância motivada pelas ações governamentais que objetivam auxiliar o combate de dois agravos importantes no País: pobreza e elevados níveis de mortalidade infantil.

Enfatizou-se que programas com condicionalidades em sua composição possuem um potencial sobre os determinantes sociais de saúde e nos cuidados primários de saúde, valendo salientar que cabe aos gestores públicos fornecerem meios para os beneficiários cumprirem com as exigências impostas por estas condicionalidades.

\section{REFERÊNCIAS}

Bassett, L. (2008). Can conditional cash transfer programs play a greater tole in reducing child undernutrition? Washington DC: World Bank, Discussion Paper № 0835.

Constituição da República Federativa do Brasil de 1988. (1998). Brasília. Recuperado em 16 abril 2016, de http://www.planalto.gov.br/CCIVIL_03/Constituicao/Constitui\%C3\%A7ao.htm

Cotta, R. M. M., \& Machado, J.C. (2013). Programa Bolsa Família e segurança alimentar e nutricional no Brasil: revisão crítica da literatura. Revista Panamericana de Salud Publica, 33(1), 54-60.

Fundo das Nações Unidas para a Infância. (UNICEF). Situação Mundial da Infância. Caderno Brasil. Brasília: UNICEF; 2008.

Gillespie, S. R., Mason, J. B., \& Martorell, R. How nutrition improves. 1996; Geneva, United Nations Administrative Committee on Coordination/ Sub-Committee on Nutrition (ACC/SCN). State-of-the-Art Nutrition Policy Discussion Paper № 15.

Guanais, F.C. (2013). The Combined Effects of the Expansion of Primary Health Care and Conditional Cash Transfers on Infant Mortality in Brazil, 1998-2010. American Journal of Public Health, 103(11), 2000-6.

Hartz, Z. M. A., Champagne, F., Leal, M.C., \& Contandriopoulos, A. (1996). Mortalidade infantil "evitável" em duas cidades do Nordeste do Brasil: indicador de qualidade do sistema local de saúde. Revista de Saúde Pública, 30(4), 310-8.

Instituto Brasileiro de Geografia e Estatística (IBGE). Departamento de população e indicadores sociais. Evolução e Perspectivas da Mortalidade Infantil no Brasil. Rio de Janeiro: IBGE; 1999. p. 45.

Instituto Brasileiro de Geografia e Estatística (IBGE). A mortalidade no Brasil no período 19802004: desafios e oportunidades para os próximos anos. Rio de Janeiro: IBGE; 2005.

Instituto Brasileiro de Geografia e Estatística (IBGE). Sobrevivência e desenvolvimento infantil. Taxa de Mortalidade Infantil. 2010. Recuperado em 15 julho, 2016, de http://teen.ibge.gov.br/biblioteca/293-teen/mao-na-roda/populacao-economia-esociedade/3371-criancas-adolescentes-e-jovens.html

Instituto Brasileiro de Geografia e Estatística (IBGE). Censo 2010: escolaridade e rendimento aumentam e cai mortalidade infantil. Rio de Janeiro: IBGE; 2012.

Instituto de Pesquisa Econômica e Aplicada. (IPEA). Objetivos de Desenvolvimento do Milênio. Relatório Nacional de Acompanhamento. Brasília: IPEA; 2014.

Mattei, L. (2010). Notas sobre programas de transferência de renda na América Latina. № 10/20010. Instituto de Estudos Latino-Americanos/Universidade Federal de Santa Catarina.

Mccoll, K. (2008). New York's road to health. British Medical Journal, 337(76620), 142-4. 
Mendes, K. D. S., Silveira, R. C. C. P., \& Galvão, C. M. (2008). Revisão integrativa: método de pesquisa para a incorporação de evidências na saúde e na enfermagem. Texto \& Contexto Enfermagem, 17(4), 758-64.

Ministério da Saúde (MS). Pacto pela redução da Mortalidade Infantil no Nordeste e na Amazônia Legal 2009-2010. ODM. Saúde Brasil. Volume III. 2010.

Ministério da Saúde (MS). 2014. ONU registra aumento da expectativa de vida no Brasil. Recuperado em 17 abril, 2016, de http://www.blog.saude.gov.br/index.php/34202-onuregistra-aumento-da-expectativa-de-vida-no-brasil

Ministério do Desenvolvimento Social e Agrário. (2013). Bolsa Família. Recuperado em 10 março, 2016, de http://mds.gov.br/assuntos/bolsa-familia

Monteiro, C. A., Conde, W. L., \& Popkin, B. M. (2002). Is Obesity Replacing or Adding to Undernutrition? Evidence from Different Social Classes in Brazil. Public Health Nutrition, 5(1A), 105-112.

Monteiro, C. A. (2003). A dimensão da pobreza, da desnutrição e da fome no Brasil. Estudos Avançados, 48(17), 7-20.

Moreira, L. M. C., Alves, C. R. L., Belisário, A. S., \& Bueno, M. C. (2012). Políticas públicas voltadas para a redução da mortalidade infantil: uma história de desafios. Revista Médica de Minas Gerais, 22(7), 48-55.

Pompeo, D. A., Rossi, L. A., \& Galvão, C. M. (2009). Integrative literature review: the initial step in the validation process of nursing diagnoses. Acta Paulista de Enfermagem, 22(4), 1-4.

Portal Brasil. Brasil retira 36 milhões da miséria extrema e bate meta da ONU para mortalidade infantil. 2014. Recuperado em 12 maio, 2016, de http://www.brasil.gov.br/centro-aberto-demidia/noticias/brasil-retira-36-milhoes-da-miseria-extrema-e-bate-meta-da-onu-paramortalidade-infantil-1

Rasella, D., Aquino, R., Santos, C. A. T., Paes-Sousa, R., \& Barreto, M. L. (2013). Effect of a conditional cash transfer programme on childhood mortality: a nationwide analysis of Brazilian municipalities. The Lancet, 382(9886), 57-64.

Rutstein, D. D., Berenberg, W., Chalmers, T.C., Child, C.G., Fishman, A.P., \& Perrin, E.B. (1976). Measuring the quality of medical care: a clinical method. New England Journal of Medicine, 294(11), 582-8.

Secretaria de Saúde/SP. Diminui o índice de mortalidade infantil no Brasil.

Recuperado em 17 julho, 2016, de http://www.saude.sp.gov.br/instituto-desaude/homepage/ultimas-noticias/diminui-o-indice-de-mortalidade-infantil-no-brasil

Senna, M. C. M., Burlandy, L., Monnerat, G.L., Schottz, V., \& Magalhães, R. (2007). Programa Bolsa Família: nova institucionalidade no campo da política social brasileira? Revista Katálysis, 10(1), 86-94.

Shei, A. (2013). Brazil's Conditional Cash Transfer Program Associated With Declines In Infant Mortality Rates. Health Affairs, 32(7), 1274-1281.

Simões, C. C. S. Perfis de saúde e de mortalidade no Brasil: uma análise de seus condicionantes em grupos populacionais específicos - Brasília: Organização Pan-Americana da Saúde, 2002.

Smith, L. C., \& Haddad, L. Overcoming Child Malnutrition in Developing Countries: Past Achievements and Future Choices. 2000; Washington, International Food Policy Research Institute, Food, Agriculture, and the Environment - Discussion Paper 30.

Stetler, C. B., Morsi, D., Rucki, S., Broughton, S., Corrigan, B., Fitzgerald, J., Giuliano, K., Havener, P., \& Sheridan, E. A. (1998). Utilization-focused integrative reviews in a nursing service. Applied Nursing Research, 11(4), 195-206.

Whttemore, R., \& Knaff, K. (2005). The integrative review: update methodology. Journal of Advanced Nursing, 52(5), 546-53.

Wolf, M. R., \& Barros Filho, A. A. (2014). Estado nutricional dos beneficiários do Programa Bolsa Família no Brasil - uma revisão sistemática. Ciência \& Saúde Coletiva, 19(5), 1331-8. 